\title{
Influence of box type and heights on movement strategies of thoracolumbar region and knee in load handling activities
}

\author{
Vivian Farahte Giangiardi', Marina Athayde Avanzi', Luciana do Socorro da Silva Valente', \\ Luciana Cristina da Cunha Bueno Silva², Ana Beatriz Oliveira², Sandra Maria Sbeghen Ferreira de Freitas', \\ Rosimeire Simprini Padula'
}

\begin{abstract}
Introduction: Handling activities which includes tasks like lifting, loading, pushing and pulling heavy loads have still been used by workers, in general, this activities are characterized for theirs no corporal limits respect; where the overload in the movements herewith theirs duration and intensity may lead to musculoskeletal diseases, which mainly affect the knee joint and thoracolumbar region of the trunk. Objective: This study intends to evaluate the movement strategies of the spine and knee joint of healthy subjects during a load manual handling course verifying the frequency of these strategies and by what they are influenced (handling high and kind of box). Methods: 42 healthy subjects participated in this study, which had to perform a manual box handling task and unload it in three highs: on the level of the floor, on trochanter's measured level and shoulder's measured level. There were utilized four kinds of boxes with variations in: size, material, and presence or not of handles. The conditions (kind of the box and high) were randomized for the subjects. The analysis was made using the unload of the boxes as a reference. Results: the results showed that the characteristics of the boxes (size, material and kind of box, with or without handles) made little influence on the strategies of knee and spine. The principal influence on the posture changes was the difference between the removals and unloads highs. Conclusions: it seems the strategies adopted by the subjects were independent of the characteristics of the load, but dependent of the task demands (i.e., handling high). Keywords: lower extremity; posture; spine; workers.
\end{abstract}

\section{INTRODUCTION}

Society is in constantly changing in relation to new technologies or the forms and working conditions. Companies from developed and developing countries, have added more technology to the manufacture of their products and replace in many cases, manual labor by automated. ${ }^{(1)}$ However, when we take into account the transfer and distribution of what is produced, handling activities involving the tasks of lifting, hold, carry, push and pull heavy loads are still widely used, ${ }^{(2)}$ and mostly do not respect the body limits. ${ }^{(3)}$

Equipment such as cars, freight elevators, forklifts, cranes and conveyor belts, it can be found to aid in the transfer of tasks, nevertheless, much of the handling activity occurs without help of these devices, so the adequacy of workers to their jobs occurs with the use of postural strategies based on their individual experiments ${ }^{(4)}$. Workers tend to use different postural strategies to do their jobs, even with under similar conditions $^{(5)}$. Lots of factors can influence the postures and movements such as the type of task, tools and furniture and may result in the appearance of Musculoskeletal disorders ${ }^{(6)}$.

The material handling tasks are an example of task, which generate musculoskeletal overload due to joint positions, which added to the duration and intensity of exercise can cause dysfunction in upper and lower limbs and the trunk. ${ }^{(7)}$ Osteoarthritis, lower back pain, femoro-patellar disorders, tendinitis, bursitis and other diseases are common to these workers. ${ }^{(7)}$

Besides, we have seen that although the upper limb be greatly affected in these cases; trunk and lower limbs, in particular the knee joint and the thoracolumbar region, are noted for receiving a higher load due to angular variations and forces generated during handling. ${ }^{(8-11)}$ Meanwhile, studies that describe the behavior of the spine and lower limbs when manipulating boxes with different characteristics, in order to relate the boxes and the behavior of the lower limbs, are still scarce compared to the presence of studies focused on upper limbs.

There are guides established in an attempt to guide the workers for handling loads ${ }^{(12-13)}$ but the shapes diversify substantially and the workers often use different methods 
from those recommended. ${ }^{(14)}$ These variations may be related to, for example, size of the loads to be handled and the type box (with or without handles), ${ }^{(15)}$ and the height that the load should be positioned. ${ }^{(16)}$

In the literature can be found many studies that describe the postural strategies used by workers in different forms of loading boxes relating experiences gained and no experience, ${ }^{(4-6,17-19)}$ showing that there are still a need for studies that seek to identify individuals strategies, in order to prevent future injuries.

Therefore, this study aimed to evaluate the knee and spine movement strategies of healthy individuals for handling loads, checking the frequency of these strategies and the factors that most influenced them, whether they are the height at which the load should be placed or the handled box model.

\section{METHODS}

\section{Study Design}

This is an observational, cross-sectional study. According to the sample calculation performed in ENE 2.0 for a 0.8 statistical power and a significance level of 0.05 were evaluate a sample of 37 individuals. Therefore, 42 volunteers were selected based on the following inclusion criteria: adults with right hand dominance, men aged between 18 to 39 years, height ranging between 1.65 and $1.75 \mathrm{~m}$, body mass between
57 and $90 \mathrm{~kg}$, and no experience in cargo handling tasks in the workplace. Were excluded individuals who presented musculoskeletal disorders of the upper limbs, trunk or lower limbs, intolerance palpation, pain, skin lesions, wastage and hypersensitivity, diseases or balance-related disorders. The study was approved by the Research Ethics Committee (CAAE - 0054.0.0135.000-07) and after being explained and presented the study objectives and procedures, all participants signed the consent and enlightened form.

\section{Materials}

For this study were used four boxes models weighing $15 \mathrm{~kg}$ each, being one of normal carton (A) without handles $(44 \times 31 \times 28 \mathrm{~cm})$, one of prototype cardboard (B) $(44 \times 31 \times 31.5 \mathrm{~cm})$ with handles $(12 \times 4 \mathrm{~cm}$ and $5 \mathrm{~cm}$ below the upper edge), one of normal plastic (C) $(55.5 \times 36 \times 31 \mathrm{~cm})$ with handles and one of prototype plastic $(36 \times 55.5 \times 31 \mathrm{~cm})$ with special handles (D) $(13 \times 4.5 \mathrm{~cm})$ (figure 1). The prototype plastic boxes and prototype cardboard were created to be different ways to get the changes in the handles, while the ordinary plastic boxes and ordinary cardboard boxes are the boxes commonly used for storage products in free fairs or locations where there is transportation of materials. It was also used a height adjustable type of shelf for unloading in so-called high and intermediate surfaces (Figure 1). To register

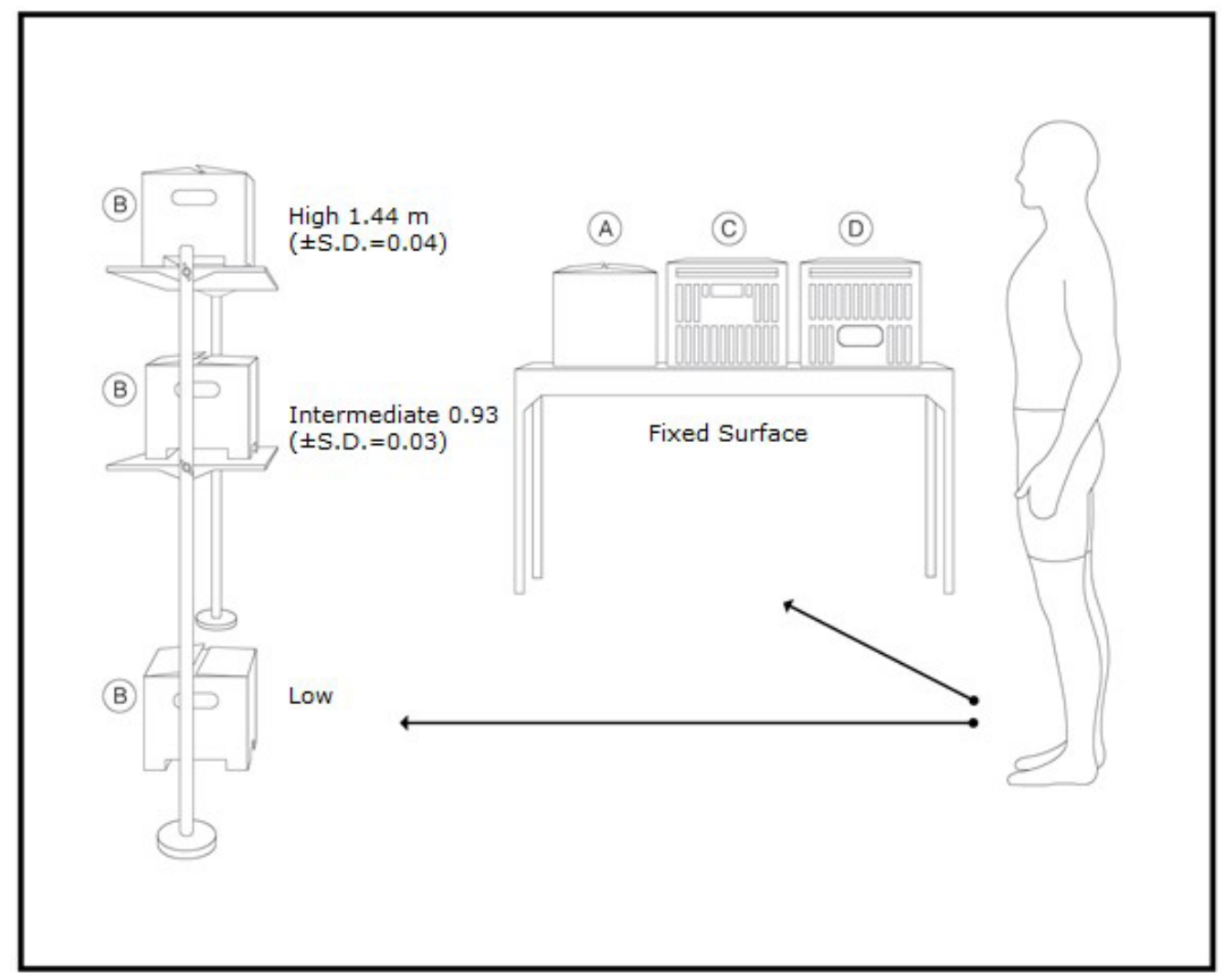

Figure 1. Layout of experimental conditions for manual handling of prototype cardboard (A), normal paper (B), normal plastics (C), prototype plastic (D) boxes. 
the task were used a digital camera Vivitar - Model DVR-518BK and tripod.

\section{Task description}

The task was simulated and aimed to reproduce the handling of boxes, with the completion of a box load path, with layout in inverted " $L$ ", to be discharged in three heights: low (at ground level), intermediate (at the height of the trochanter) and high (at shoulder height) (figure 1). Participants should take a box of a standard height ("withdrawal") and discharges it ("discharge") in one of the three heights, wherein the sequence of handling of four box designs for the three heights were determined at the beginning of the collection of each participant and randomly assigned to each individual. Participants did not know the weight of the boxes that they would handle, and they were not instructed on how they should pick up the boxes. Only the initial and final positions were demonstrated to them, which allowed free movement of handling between them.

The path was filmed for later carried out the analysis of knee and thoracolumbar region movement strategies, at the time of discharge of each box in different heights.

\section{Criteria for motion analysis}

The analysis of movements of the knee and spine, in order to define the strategies used for discharge of the boxes, was performed by classifying amplitude bands, according to parameters used in observational methods of risk analysis. ${ }^{(20)}$ The analysis was carried out during cargo placing (discharge) on the shelf at different heights (low, moderate, high). The handling path and the removal step of the fixed height box that was adjustable to the individual's waist were not considered for analysis. The part of the filming used for the analysis was the time when the participant deposited the box on the shelf ("discharge"). At this time the image has been "frozen" for analysis and determination of the range of motion.

The criteria used for the knee movement classification were based on the angle between the posterior portion of the thigh, and the posterior portion of the leg ${ }^{(21)}$ being: a) partial flexion with angulation up to $90^{\circ}$ or higher in one or both knees (right or left); b) full flexion with angulation up to $90^{\circ}$ or lower of one or both knees (right or left); c) a neutral position with no type of knee flexion (partial or full) keeping the extent to $180^{\circ}$; d) knee flexion position (partial or full), or neutral, associated with plantar flexion of the ankle; e) knee in a neutral position (maintaining the extension to $180^{\circ}$ ), unilaterally associated with plantar flexion of the ankle.

The criteria used for the spine movement classification were based on the angle between the trunk and the anterior portion of the thigh ${ }^{(21)}$ being: a) partial flexion with angulation of $30^{\circ}$ to $90^{\circ}$ of the truck with the thigh; b) full flexion with angulation greater than $90^{\circ}$ of the truck with the thigh; c) extension position with angulation up to $30^{\circ}$ of posterior displacement; d) neutral position without flexion (full or partial)or extension, with maintenance of the trunk in an angulation of $180^{\circ}$.

A sheet containing all classifications of amplitude ranges of knee joints and spine was used to check each volunteer in the handling of each type of box in different heights.

\section{Statistical analysis}

The SPSS 17.0 program was used for all analyzes. The classification of the movement angles in all handling heights using each one of the 4 boxes are presented as absolute and relative frequencies. The chi-square and Fisher's exact tests were used, with $5 \%$ significance level, to assess the association between height and type of box with the postures of knee and spine.

\section{RESULTS}

Table 1 has the results of normal cardboard box in the three handling heights. The position of both knees in full flexion in Low height prevailed (73.8\%), while the Intermediate height in neutral position of the left knee was higher $(85.7 \%)$ followed by partial flexion of the right knee (61.9\%). At the High height, the frequency was greater in neutral position of the left knee $(54.8 \%)$, followed by neutral position of the right knee (47.6\%). In sequence, the unilateral plantar flexion of the ankle was higher in the left side (39.0\%) compared to the right side (31.0\%, Table 1$)$.

Table 2 shows the results for prototype cardboard box, there is predominance in Low height of both knees in full flexion (76.2\%). For Intermediate height the neutral position of the left knee was the most used (81.0\%), and right knee was more used to the partial flexion (54.8\%) followed by its neutral position (38.1\%). At the high height the greater frequencies were neutral to the left knee (48.8\%), followed by the unilateral ankle plantar flexion (41.5\%); in sequence the right knee in unilateral ankle plantar flexion (39.0\%) and knee in a neutral position (36.6\% - Table 2).

Table 3 presents the data for normal plastic box. At low height in both knees ( $R$ and $L$ ) the partial flexion (59.5\% and $57.1 \%$, respectively) was more frequent. The predominated position in Intermediate height was the neutral position in the left knee (78\%), followed by partial flexion (41.2\%) and neutral position (48.8\%) of the right knee. During the High height occurred more frequently the neutral position of the left knee (62.5\%), followed by unilateral ankle plantar flexion to the right ( $45.0 \%$ - Table 3$)$.

In Table 4 are the results for the prototype plastic box. For Low height there was more frequency (66.7\%) for both knees in full flexion. In the intermediate height, the most frequently found refers to the neutral position of the left knee (81.0\%), followed by neutral (47.6\%) and partial flexion (45.2\%) of right knee. For the High height occurred more frequently the 
Table 1. Frequency of occurrence \% ( $n$ ) of the knee positions (right and left) during discharge of the normal cardboard box for low, intermediate and high heights.

\begin{tabular}{|c|c|c|c|c|c|c|}
\hline \multirow{3}{*}{ Knee } & \multicolumn{6}{|c|}{ NORMAL CARDBOARD } \\
\hline & \multicolumn{2}{|c|}{ Low (ground) } & \multicolumn{2}{|c|}{ Intermediate (trochanter) } & \multicolumn{2}{|c|}{ High (shoulder) * } \\
\hline & RK & LK & RK & LK & RK & LK \\
\hline PF & $26.2(11)$ & $26.2(11)$ & $4.8(2)$ & $4.8(2)$ & - & - \\
\hline $\mathrm{FF}$ & $73.8(31)$ & $73.8(31)$ & $61.9(26)$ & $4.8(2)$ & $9.5(4)$ & $2.4(1)$ \\
\hline N & - & - & $28.6(12)$ & $85.7(36)$ & $47.6(20)$ & $54.8(23)$ \\
\hline$P F+P$ & - & - & - & $2.4(1)$ & $9.5(4)$ & $2.4(1)$ \\
\hline $\mathrm{N}+\mathrm{P}$ & - & - & - & $2.4(1)$ & - & - \\
\hline $\mathrm{U}+\mathrm{P}$ & - & - & $4.8(2)$ & - & $31.0(13)$ & $39.0(16)$ \\
\hline Spine & - & - & & - & - & - \\
\hline N & - & - & 100.0 & - & 26.8 & - \\
\hline Ex & - & - & - & - & 73.2 & - \\
\hline $\mathrm{PF}$ & 73.8 & - & - & - & - & - \\
\hline $\mathrm{FF}$ & 26.2 & - & - & - & - & - \\
\hline
\end{tabular}

Knee: PF - partial flexion; FF - full flexion; N - neutral; PF + P - partial knee flexion associated with plantar flexion of the ankle; N+P - knee in a neutral position associated with plantar flexion of the ankle; U+P - knee in a neutral position (both) associated with unilateral plantar flexion of the ankle. * technical problems, results for 41 participants, 1 participant in this condition could not perform the analysis. Spine: $\mathrm{N}$ - neutral posture; Ex - extension; PF - partial flexion; FF - full flexion. ${ }^{*}$ technical problems, results for 41 participants, 1 participant could not perform the analysis in this condition.

Table 2. Frequency of occurrence \% ( $\mathrm{n}$ ) of the knee (Right and Left) and spine positions during the discharge of the prototype cardboard box for low, intermediate and high heights.

\begin{tabular}{|c|c|c|c|c|c|c|}
\hline \multirow{3}{*}{ Knee } & \multicolumn{6}{|c|}{ PROTOTYPE CARDBOARD } \\
\hline & \multicolumn{2}{|c|}{ Low (ground) } & \multicolumn{2}{|c|}{ Intermediate (trochanter) } & \multicolumn{2}{|c|}{ High (shoulder)* } \\
\hline & RK & LK & RK & LK & RK & LK \\
\hline $\mathrm{PF}$ & $23.8(10)$ & $23.8(10)$ & $54.8(23)$ & $14.3(6)$ & $12.2(5)$ & $7.3(3)$ \\
\hline $\mathrm{FF}$ & $76.2(32)$ & $76.2(32)$ & - & - & - & - \\
\hline $\mathrm{N}$ & - & - & $38.1(16)$ & $81.0(34)$ & $36.6(15)$ & $48.8(20)$ \\
\hline $\mathrm{PF}+\mathrm{P}$ & - & - & - & - & $12.2(5)$ & $2.4(1)$ \\
\hline $\mathrm{N}+\mathrm{P}$ & - & - & - & - & - & - \\
\hline$U+P$ & - & - & $7.1(3)$ & $4.8(2)$ & $39.0(16)$ & $41.5(17)$ \\
\hline Spine & - & - & - & - & - & - \\
\hline $\mathrm{N}$ & - & - & $100.0(42)$ & - & $19.5(8)$ & - \\
\hline Ex & - & - & - & - & $80.5(33)$ & - \\
\hline $\mathrm{PF}$ & $73.8(30)$ & - & - & - & - & - \\
\hline $\mathrm{FF}$ & $26.2(12)$ & - & - & - & - & - \\
\hline
\end{tabular}

Knee: PF - partial flexion; FF - full flexion; $\mathrm{N}$ - neutral; $\mathrm{PF}+\mathrm{P}-$ partial knee flexion associated with plantar flexion of the ankle; $\mathrm{N}+\mathrm{P}-\mathrm{knee}$ in a neutral position associated with plantar flexion of the ankle; U+P - knee in a neutral position (both) associated with unilateral ankle plantar flexion. Spine: $\mathrm{N}-$ neutral posture; Ex - extension; PF - partial flexion; FF - full flexion. * technical problems, results for 41 participants, 1 participant could not perform the analysis in this condition.

neutral position of the left knee $(66.7 \%)$, followed by neutral position of the right knee ( $50 \%$ - Table 4$)$.

Regarding the results of the postures used in the spine movement during the discharge of boxes for Low height, the boxes: Cardboard Normal (73.8\%), Prototype Cardboard (73.8\%) and Prototype Plastic (66.7\%) showed a higher frequency of partial flexion of the spine; while the Normal Plastic box presented more frequently $(56.1 \%)$ of full flexion of the spine. For intermediate height in all cases the prevalence was the neutral position of the spine (100.0\%). For High height in all cases occurred more frequency of the extension of the spine $(73.2 \%, 80.5 \%, 97.5 \%$ and $92.9 \%$, respectively for the Normal Cardboard, Prototype Cardboard, Normal Plastic and Prototype Plastic boxes).

Analyzes of association showed that just the Heights of handling influenced the postures of the spine and knees. There 
Table 3. Frequency of occurrence \% $(n)$ of the knee (Right and Left) and spine positions during discharge of the normal plastic box for low, intermediate and high altitudes.

\begin{tabular}{|c|c|c|c|c|c|c|}
\hline \multirow{3}{*}{ Knee } & \multicolumn{6}{|c|}{ NORMAL PLASTIC } \\
\hline & \multicolumn{2}{|c|}{ Low (ground) } & \multicolumn{2}{|c|}{ Intermediate (trochanter)* } & \multicolumn{2}{|c|}{ High (shoulder)** } \\
\hline & RK & LK & RK & LK & RK & LK \\
\hline $\mathrm{PF}$ & $59.5(25)$ & $57.1(24)$ & $41.2(16)$ & $19.5(8)$ & $12.5(5)$ & $7.5(3)$ \\
\hline $\mathrm{FF}$ & $40.5(17)$ & $40.5(17)$ & - & - & - & - \\
\hline $\mathrm{N}$ & - & $2.4(1)$ & $48.8(20)$ & $78(32)$ & $30.0(12)$ & $62.5(25)$ \\
\hline $\mathrm{FP}+\mathrm{P}$ & - & - & - & $2.4(1)$ & $12.5(5)$ & - \\
\hline $\mathrm{N}+\mathrm{P}$ & - & - & - & - & - & - \\
\hline$U+P$ & - & - & - & - & $45(18)$ & $30.0(12)$ \\
\hline Spine & - & - & - & - & - & - \\
\hline $\mathrm{N}$ & $2.4(1)$ & - & $100.0(41)$ & - & $2.5(1)$ & - \\
\hline Ex & - & - & - & - & $97.5(39)$ & - \\
\hline $\mathrm{PF}$ & $41.5(18)$ & - & - & - & - & - \\
\hline $\mathrm{FF}$ & $56.1(23)$ & - & - & - & - & - \\
\hline
\end{tabular}

Knee: $\mathrm{PF}$ - partial flexion; FF-full flexion; $\mathrm{N}$ - neutral; PF+P - partial knee flexion associated with plantar flexion of the ankle; $\mathrm{N}+\mathrm{P}-\mathrm{knee}$ in a neutral position associated with plantar flexion of the ankle; U+P - knee in a neutral position (both) associated with unilateral plantar flexion of the ankle. Spine: $\mathrm{N}-$ neutral posture; Ex - extension; PF - partial flexion; FF - full flexion. * technical problems, results for 41 participants, 1 participant could not perform the analysis in this condition. ${ }^{* *}$ technical problems, results for 40 participants, 2 participant could not perform the analysis in this condition

Table 4. Frequency of occurrence \% ( $\mathrm{n}$ ) of the knee (Right and Left) and spine positions during discharge of the prototype plastic box for low, intermediate and high heights.

\begin{tabular}{|c|c|c|c|c|c|c|}
\hline \multirow{3}{*}{ Knee } & \multicolumn{6}{|c|}{ PROTOTYPE PLASTICS } \\
\hline & \multicolumn{2}{|c|}{ Low (ground) } & \multicolumn{2}{|c|}{ Intermediate (trochante) } & \multicolumn{2}{|c|}{ High (shoulder) } \\
\hline & RK & LK & RK & LK & RK & LK \\
\hline $\mathrm{PF}$ & $33.3(14)$ & $33.3(14)$ & $45.2(19)$ & $16.7(7)$ & $14.3(6)$ & $2.4(1)$ \\
\hline $\mathrm{FF}$ & $66.7(28)$ & $66.7(28)$ & - & - & - & - \\
\hline $\mathrm{N}$ & - & - & $47.6(20)$ & $81.0(34)$ & $50.0(21)$ & $66.7(28)$ \\
\hline$P F+P$ & - & - & $2.4(1)$ & - & $7.1(3)$ & $2.4(1)$ \\
\hline $\mathrm{N}+\mathrm{P}$ & - & - & - & - & - & - \\
\hline$U+P$ & - & - & $4.8(2)$ & $2.4(1)$ & $28.6(12)$ & $28.6(12)$ \\
\hline Spine & - & - & - & - & - & - \\
\hline $\mathrm{N}$ & - & - & $100.0(42)$ & - & $7.1(3)$ & - \\
\hline Ex & - & - & - & - & $92.9(39)$ & - \\
\hline PF & $66.7(28)$ & - & - & - & - & - \\
\hline TF & $33.3(14)$ & - & - & - & - & - \\
\hline
\end{tabular}

Knee: PF - partial flexion; FF - full flexion; $\mathrm{N}$ - neutral; PF+P - partial knee flexion associated with plantar flexion of the ankle; $\mathrm{N}+\mathrm{P}-$ nee in a neutral position associated with plantar flexion of the ankle; $\mathrm{U}+\mathrm{P}-$ knee in a neutral position (both) associated with unilateral ankle plantar flexion. Spine: $\mathrm{N}-$ neutral posture; Ex-extension; $\mathrm{PF}-$ partial flexion; $\mathrm{FF}-$ full flexion.

was a positive association between the discharge of the box to the lower height $(p<0.001)$ with the positions of the spine, as well as the right and left knees, regardless of the type of handled boxes.

\section{DISCUSSION}

This study aims to evaluate knee and spine postures used by healthy individuals during manual unloading of boxes in order to analyze the frequency and the factors that influence the use of these postures, in this case the height of the handling and the type of box.

When analyzing the postural strategies used in the discharges, because of the variance in the final heights, it was showed a relation between the knees, spine and final height. At Low height individuals used knee and spine flexion to be able to carry out the discharge, as the height increased the knees and the spine tended to extend, causing in the Intermediate height, the reach of a neutral posture for the 
knees and use the upright posture of the spine. At shoulder height, corresponding to the higher in the path, knees shifted from neutral posture to an extension in posture, which in most of the cases has caused plantar flexion and the concentration of the subject's body weight at the tip of foot, accompanied by the extension of the spine.

Those results show the great influence that the height causes in the knees and spine posture strategies, and the small influence of the type and size of the box.

According to the study realized by Gagnon et. al., ${ }^{(4)}$ which investigated the appearance of lesions and their influence, were observed that individuals who performed higher knee flexions to handling activities, have higher amounts of loads acting on the spine and knee. ${ }^{(4)}$ Furthermore, another study by Gagnon et. al. ${ }^{(20)}$ demonstrated that the knee flexion influences the distribution of strength moments between vertebrae L5 / S1 of the column and the knee joint, affecting the stability of individuals. It has been proven that slight flexion of the knee makes the torque, which act on it, larger at the expense of decreased stability body. ${ }^{(20)}$ Thus, the discharge of loads at lower heights that require greater flexion of the knee will cause major instabilities, overloads and increased risk of the appearance of injuries.

This was also demonstrated by Kingma et. al. ${ }^{(22)}$, which after analysis of different techniques for manual lifting loads, showed that the smaller the bending amplitudes performed by the knee and lumbar spine, the better the lifting is performed and the smaller is the risk in the appearance of injuries. ${ }^{(22)}$

Studies by Padula et. al. ${ }^{(16.23)}$ described the movements of the spine in healthy, sedentary and workers subjects who had or not musculoskeletal symptoms noting that the height of the surface to which individuals aimed at discharging the load exerted a significant influence in the spine positions. When subjects aimed higher Heights were observed higher spine extension movements, but when individuals aimed lower altitudes, were observed higher flexion movements. It was confirmed that the spine extension movements requires a greater time spent than flexion movements, besides requiring greater efforts for maintaining the position making the amount of overhead act longer and increasing the risk of injury. ${ }^{(16.23 .24)}$

In the guides were performed guidelines for handling loads, in which the main guidelines were to reduce the risk of appearance of injuries in the perception of bodily limitations of each individual, avoiding the repetitive work, seeking to promote the handling of loads closest to the body and the use of tools that can help in this handling, as well as the formulation of strategies which showed the focus of concern in the size and type of material to be handled.

This study showed that to reduce the risk of appearance of injuries, the size and type of box will only be influencing if the removal and discharge times are equal, that is, do not exhibit variance. But when the manual loading of cargo is done with variance in heights the main influence on the appearance of injuries is related to the final discharge height.

Workers who use the manual loading of cargo have the presence of local discharges and withdrawals with varying heights, and with the consequent occurrence of knee and spine injuries, which can make it difficult and even impossible the work and daily life activities. To avoid injuries the main measure to be taken is not only related to the change in the type and size of box used, but with the change in the withdrawal and discharge of boxes to be loaded.

Some limitations occurred during the analysis of the videos related to the Position of the researcher during the collection in front of the participants, making it impossible to check and analysis of some postures of knee and spine, however, it was mentioned in the footnotes of the tables. Another limitation may be related to the available space for the realization of the path that was restricted to the area between the fixed and adjustable surfaces, which may have influenced some positions and adjustments.

\section{CONCLUSION}

The strategies of the spine and knee, in relation with the discharges to the strategies tendency, was related to the final height of the surface, and at low height knees and the spine was presented more flexed, as the height increased the knees and the spine tends to extend until the reach of a neutral posture. In high height was promoted the occurrence of the lifting of the ankles concentrating the weight on tiptoes, generating the extension of the knees and spine.

Thus, the study shows the great influence of heights and the little influence of the type and size of the boxes in the knee and spine strategies during discharge of cargo.

\section{AUTHOR'S CONTRIBUTIONS:}

We confirm that VFG, LSSV, LCCBS, ABO and contributed with conception of the study. LSSV and LCCBS collected data. Data analysis and interpretation were conducted by VFG, LSSV, SMSFF and RSP. All authors drafting and approval of the final version the article.

\section{CONFLICT OF INTEREST:}

No potential conflict of interest was reported by the authors.

\section{AUTHOR DETAILS}

${ }^{2}$ Department of Physical Therapy, Universidade Federal de São Carlos, São Carlos, SP, Brazil

\section{REFERENCES}

1. Dempsey PG, Mathiassen SE. On the evolution of task-based analysis of manual materials handling, and its applicability in contemporary ergonomics. Appl Ergon. 2006; 37:33-43.

2. Zurada J. Classifying the risk of musculoskeletal low back disorders due to manual material handling tasks. Expert Systems with Application. 2012; 39:1125-134.

3. Bureau of Labor Statistics. Nonfatal Occupational Injuries and IIInesses Requiring Days Away from Work, 2012. 
4. Gagnon M. Box tilt and knee motion in manual lifting: two differential factors in expert and novice workers. Clin Biomech. 1997; 12(718):419-28,

5. Coury HG, Padula RS. Trunk movements and load support strategy in simulated handling tasks carried out by workers with and without musculoskeletal symptoms. Clin Biomech. 2002; 17:309-11.

6. Madeleine P, Lundager B, Voigt M, Arendt-Nielsen L. Standardized low-load repetitive work: evidence of different motor control strategies between experienced workers and a reference group. Appl Ergon. 2003; 34:533-42.

7. Simoneau S, ST-Vincent M, Chicoine D. Work Related Musculoskeletal Disorders (WMSDs), a better understanding for more effective prevention. Association paritari por la santé at la sécurité du travail. Institute de recherché Robert-Sauvé en santé et en sécurité du travail du Quebéc, 2003.

8. Davis KG, Marras WS. The effects of motion on trunk biomechanics - Clin Biomech. 2000; 15:703-17.

9. Junior MT, Fancello EA, Roesler CRM, More ADO. Simulação numérica tridimensional da mecânica do joelho humano. Acta Ortopédica Brasileira. 2008; 17( 2):18-23.

10. Keyserling WM, Brouwer $M$, Silverstein BA. A checklist for evaluating ergonomic risks factors resulting for awkward postures of the legs, trunk and neck. Int J Ind Ergon. 1992; 9 (4):283-301.

11. Marras WS, Lavender SA, Leurgans SE, Fathallah FA, Ferguson SA, Allread WG, Rajulu SL. Biomechanical risk factors for occupationally related low back disorders. Ergonomics. 1995; 38(2): 377-410.

12. The National Institute for Occupational Saffety and Healthy (NIOSH), 2007. Ergonomic Guidelines for Manual Material Handling. Disponível em: http://www.cdc.gov/niosh/docs/2007-131/pdfs/2007-131.pdf

13. Occupational Healthy and Safety Administration (OSHAS), 2002. Material Handling and Storage. Disponível em: http://www.osha.gov/Publications/ osha2236.pdf
14. Zhou J, Dai B, Ning X. The assessment of material handling strategies in dealing with sudden loading: influences of foot placement on trunk biomechanics. Ergonomics. 2013; 56(10):1569-76.

15. Jung HS. A survey of the optimal handle position for boxes with different sizes and manual handling positions. Appl Ergon. 2010; 41:115-22.

16. Padula RS, Gil Coury HJC - Sagittal trunk movements during load carrying activities: a piloty study. Int J Ind Ergon. 2003; 32:181-8.

17. Gagnon M, Plamondon A, Gravels D, Lortie IM. Knee movement strategies differentiate expert from novice workers in asymmetrical manual materials handling. J Biomech. 1996; 29(11):1445-453.

18. Gagnon M. Ergonomic identification and biomechanical evaluation of workers strategies and their validation in a training situation: Summary of research. Clin Biomechanics. 2005, 20: 569-80

19. Simic M, Hinman RS, Wrigley TV, Bennell KL, Hunt MA. Gait Modification strategies for altering medial Knee Joint Load: A Systematic Review. Arthritis Care Res, American College of Rheumatology. 2010; 63( 3): 405426,

20. Gagnon M, Delisle A, Desjardins P. Knee flexion and base of support in asymmetrical handling: effects on the worker's dynamic stability and the moments of the L5/SI and knee joints. Clin Biomechanics. 1998; 13:506514.

21. Hall SJ. Biomecânica Básica. Ed. Guanabara Koogan. 4o edição. 2005.

22. Kingma I, Faber GS, van Dieën JH. How to lift a box that is too large to fit between the knees. Ergonomics. 2010; 53(10):1228-1238.

23. Padula RS, Oliveira AB, Barela AM, Barela JA, Gil Coury HJC. Are the anticipatory trunk movements occurring during load-carrying activities protective or risky? Int J Ind Ergon. 2008; 38:298-306.

24. Zhou J, Ning X, Nimbarte AD, Dai F. The assessment of material-handling strategies in dealing with sudden loading: the effect of uneven ground surface on trunk biomechanical responses. Ergonomics. 2015; 58(2):259267. 